\title{
Unique Filtering and Wavelength-Stabilization through FBG in WDM/DWDM Networks
}

\author{
Warsha Balani \\ M.Tech. Student \\ BIST, Bhopal \\ M.P. India
}

\author{
Manish Saxena \\ Assistant Professor \\ BIST, Bhopal \\ M.P. India
}

\begin{abstract}
Fiber Bragg gratings (FBG's) have emerged as important components in a variety of light wave applications. Their unique filtering properties and versatility as in-fiber devices is illustrated by their use in wavelength-stabilized device, This new design is applicable in high speed DWM PON architecture and filter all impairments incorporated with channels.
\end{abstract}

\section{General Terms}

Optisystem Software

\section{Keywords}

EDFA, gain flatness, Fiber Bragg grating (FBG), FWM, WDM

\section{INTRODUCTION}

LIGHTWAVE networks enable high-capacity interconnections among producers and consumers of information located at most places on the earth. Demand for increased bandwidth resulting from new services and users have stimulated innovations in the light wave industry, including the intriguing prospect of directly connecting all network users to gigabit/s optical links. Allocating communication channels to partitions in the optical frequency spectrum has established wavelength division multiplexed transmission as a conventional method of increasing capacity and enables optical methods of implementing network functions. Commercial point-to-point WDM light wave systems operating in the $1550-\mathrm{nm}$ wavelength region may have 8,16 , and more channels, each carrying 2.5 or $10 \mathrm{~Gb} / \mathrm{s}$ traffic and proposals of finer granularity, e.g., 64 channels at $622 \mathrm{Mb} / \mathrm{s}$ are appearing. Furthermore, the traditional point topoint link will be supplanted with networks that include optical add/drop or cross-connect capability. In local access, information delivery to the subscriber residence is now predominately through copper wire or wireless connections; direct links with optical fibers in passive optical networks (PON's) is emerging as a competing technology. Accompanying this evolution of the optical network infrastructure, with its emphasis on WDM transmission, is the proliferation of new optical devices. Discoveries in optical transmitters, amplifiers, frequency converters, filters, and multiplexers enable new ways to generate, condition, and detect light. Fiber Bragg grating (FBG) filters [1]-[4] have received attention for their versatility and unique filtering capabilities. $\mathrm{n}$ this paper we examine several familiar light wave applications of the FBG such as fiber lasers and dispersion compensators as well as novel uses in devices including wavelength converters and phase conjugators. The diversity of its uses in light wave communication systems may result in the FBG becoming a ubiquitous and necessary element in equipment located throughout the network from the central office to the home. Indeed, FBG's are now used in commercially available add/drop multiplexers and wavelength stabilized pump lasers. Before discussing the applications of FBG's, a tutorial introduction to grating theory is included in order to improve understanding and clarify grating design. We ask that authors follow some simple guidelines. In essence, we ask you to make your paper look exactly like this document. The easiest way to do this is simply to download the template, and replace the content with your own material.

\section{THEORY}

The study of wave interactions with periodic structures[5] has yielded convenient methods of analysis. Gratings have been investigated in classical optical structures and the results have been extended to gratings in waveguides such as those in distributed feedback lasers, acousto-optic modulators and filters, and other diffractive waveguide devices. The FBG is readily analyzed by these methods, including coupled mode and transfer matrixes analyses [6], [7]; here, we briefly describe the coupled mode analysis. More complete approaches include other effects such as radiation mode coupling and absorptive losses in the grating and are required when considering actual design and implementation of fiber gratings. The two "modes" of the waveguide Bragg grating are the counter-propagating waves in the fiber which are coupled through the grating reflection. In the absence of the grating, these partial dependence of the polarization currents for the forward traveling wave are proportionalexp $(\mathrm{jz} \beta)$.Perturbations by a grating having spatial period $\Lambda$ create new sidebands that result in wave coupling, i.e.,

$\exp (\mathrm{j} \beta \mathrm{z}) \cos \left(\frac{2 \pi \mathrm{z}}{\Lambda}\right)=\frac{1}{2}\left\{\exp \left[\mathrm{j}\left(\beta-\frac{2 \pi}{\Lambda}\right) \mathrm{z}\right]+\exp [\mathrm{j}(\beta+\right.$ $\left.\left.\left.\frac{2 \pi}{\Lambda}\right) \mathrm{z}\right]\right\} \ldots \ldots . .1$

and mode coupling to the backward coupling waveexp( $(-\mathrm{jz} \beta)$, described by the first term on the righthandside, occurs when $-\beta=\left(\beta-\frac{2 \pi}{\Lambda}\right)$ [8].Then the forward-propagating wave is reflected (coupled) into the backward-propagating wave .Coupled mode equations are readily derived upon substitution of the grating refractive index function into the wave equation 
$\frac{\mathrm{d}^{2} \mathrm{E}}{\mathrm{d} \xi^{2}}+\left[\frac{\beta \mathrm{n}(\xi}{\mathrm{k}_{0} \mathrm{n}_{0}}\right]^{2} \mathrm{E}(\xi)=0$

Where $\xi=\mathrm{K}_{0} \mathrm{z}$ is the normalized axial dimension and $\mathrm{n}(\xi) / \mathrm{n}_{0}=1+\sigma(\xi)+2 \mathrm{~h}(\xi) \cos \left[2 \xi+2 \phi(\xi)\right.$. Here $\mathrm{n}_{0}$ is the effective index of the propagating waves. andk $\mathrm{k}_{0}=\mathrm{w}_{0} \mathrm{n}_{0} /$ cis the nominal Bragg wave number; for constant

\section{THEORY OF FWM}

The origin of FWM process lies in the nonlinear response of bound electrons of a material to an applied optical field. In fact, in order to understand the FWM effect, consider a WDM signal, which is sum of $n$ monochromatic plane waves. The electric field of such signal can be written as

$$
E=\sum_{P=1}^{n} E_{p} \cos \left(w_{p} t-K_{p} z\right) \ldots .3
$$

Then the nonlinear polarization is given by

For this case $P_{n l}$ takes the form as

$$
\mathrm{P}_{\mathrm{nl}}=\varepsilon_{0} \chi^{3} \mathrm{E}^{3} \ldots \ldots 4
$$

$$
\begin{gathered}
P_{n l}=\varepsilon_{0} \chi^{3} \sum_{p=1}^{n} \sum_{q=1}^{n} \sum_{r=1}^{n} E_{p} \cos \left(w_{p} t-k_{p} t\right) E_{p} \cos \left(w_{p} t\right. \\
\left.-t_{p} z\right) E_{r} \cos \left(w_{r} t-k_{r} z\right) \ldots 5
\end{gathered}
$$

The reason behind this phase mismatch is that, in real fibers $\mathrm{k}(3 \omega) \neq 3 \mathrm{k}(\omega)$ so any difference like $(3 \omega-3 \mathrm{k})$ is called as phase mismatch. The phase mismatch can also be understood as the mismatch in phase between different signals traveling within the fiber at different group velocities. All these waves can be neglected because they contribute little. The last term represents phenomenon of four-wave mixing [3].

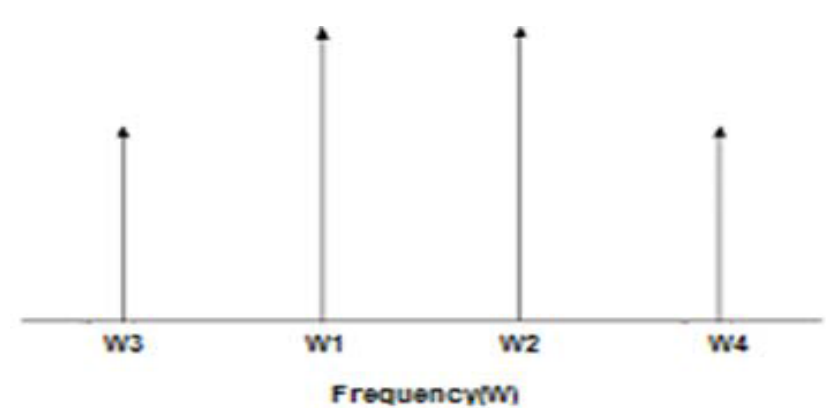

Fig.1:FWM of two wave $\omega 1$ and $\omega 2$
Fig.1. FWM of two wave $\omega 1$ and $\omega 2$

Figure 1 shows a simple example of mixing of two waves at frequency $\omega 1$ and $\omega 2$. When these waves mixed up, they generate sidebands at $\omega 3$ and $\omega 4$ such that $(\omega 1+\omega 2=\omega 3+\omega 4)$ [8]. Similarly, three co-propagating waves will create nine new optical sideband waves at frequencies given by Eq. (5). These sidebands travel along with original waves and will grow at the expense of signal-strength depletion. In general for $\mathrm{N}$ wavelengths launched into fiber, the number of generated mixed products $\mathrm{M}$ is, $\mathrm{M}=(\mathrm{N} 2 / 2)(\mathrm{N} 1)$

.6

\section{EXPERIMENTAL SETUP}

The Experimental setup is designed for the demonstration of the WDM-PON ring architecture from the OLT to the ONU. In the proposed model we have used 4 channels at $10 \mathrm{~Gb} / \mathrm{s}$ to convey the information from $\mathrm{CO}$ to $\mathrm{ONU}$ using FBG as router. A tunable laser source provides a $\mathrm{CW}$ probe signal at 193.1 to 193.4. The CW probe signal is fed to an optical mux, and then launched into SSMF before being sent to circulator, which emulate at the $\mathrm{CO}$ and the remote node, respectively. At the ONU side, we have a FBG followed by an circulator to simulate down-stream signals. The FBG is to allow tuning the wavelength of the received signal and circulator is used to isolate to mixed up forward \& backward signal thus to study the ring based operation in the proposed scheme. Downstream NRZ data modulation format running at $10 \mathrm{~Gb} / \mathrm{s}$ with a pseudo-random binary sequence(PRBS) length of $2^{7}-$ 1 bits. The global parameters are set as sampling rate at 64 $\mathrm{GHz}$, samples/bit is 64 and total sequence length is 8192 .

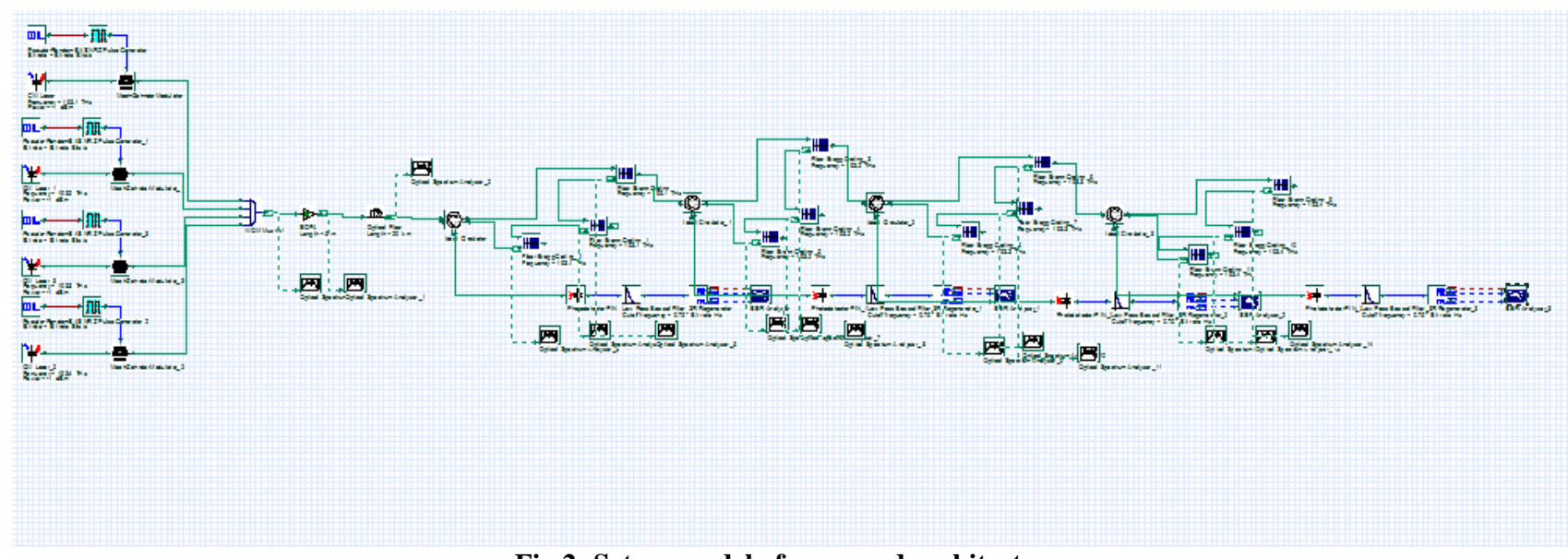

Fig 2: Set up model of proposed architecture 


\section{RESULT \& DISCUSSION}

Wavelength Spectrum of Multiplexed Data at CS: The spectrum of the multiplexed signal for these 4 signals for downlink is shown in Fig. 3 Which shows the wavelength $(\mathrm{THz})$ versus power(in $\mathrm{dBm}$ ) graph. Figure concludes that we are getting the peak powers at frequency ranging from 193.1 THz 193.4 THz. Wavelength $193.1 \mathrm{THz} 193.4 \mathrm{THz}$ represents the data signals. In this design CS is transmitting the signal from at $10 \mathrm{~Gb} / \mathrm{s}$ in the entire conventional band.

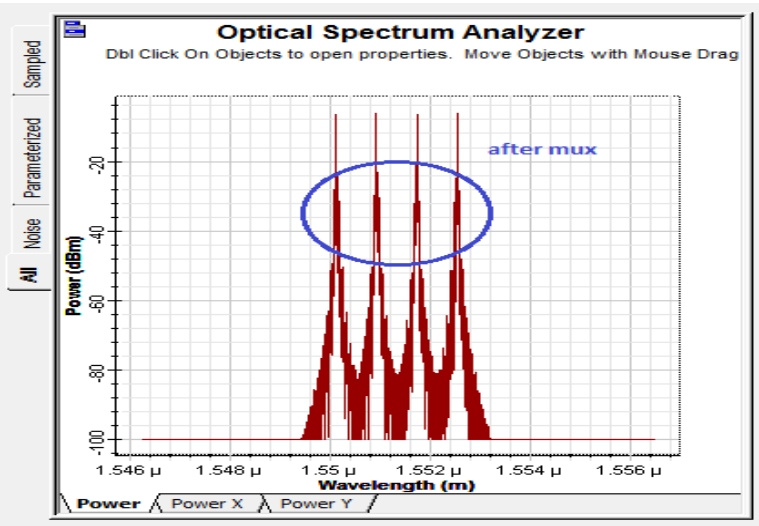

Fig 3: Received Signals after Fiber

The undersigned Figure 4 represents the spectrum plot for the multiplexed signals after traveling the $20 \mathrm{~km}$ distances. Its clear that some noise spikes are added with the data signals. but we are getting equal peak power at wavelengths at which we have modulated our signals i.e. from 193.1 to $193.4 \mathrm{THz}$. Along with these frequency range we are also getting a extra range of the frequency wings being demonstrated with the blue circle which is the result of the four wave mixing effects ,which is major issue in optical communication.

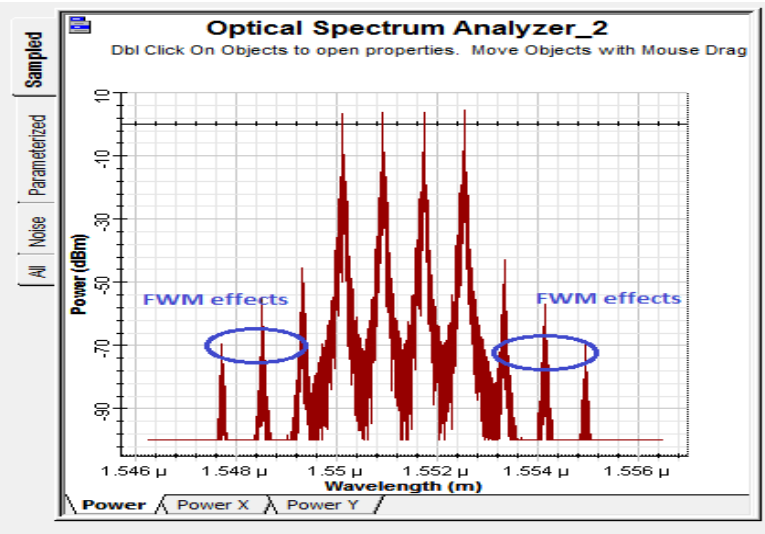

Fig 4: Spectrum plot for the multiplexed signals after traveling the $20 \mathrm{~km}$ distances

when the signal passes through the optical fiber the noise gets merge into the signal to the uttermost level. The fig 4 shows the multiplexed signal received after the signal passage through the optical fiber. The blue circle shape shows the noise level. The level of the noise is ranging from the -70 to that of $-45 \mathrm{dBm}$. These noise are easily filter from the signals at the receiver ends with the help of the FBG filter.

The filtered received frequency $193.1 \mathrm{THz}$ is clearly demonstrated in the following fig. It is clearly shows that there is no unwanted signal along-with original signal.

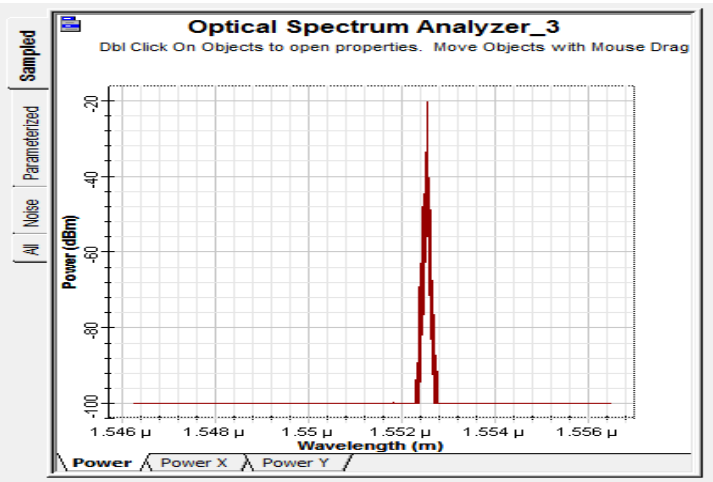

Fig 5: Filtered received frequency 193.1 THz

Analytical study based on Q Factor to the Input-Power .The output noise is the vital parameter in the any type of the analysis. In fig the graphical analysis is done between the $\mathrm{Q}$ factor vs. various input power. As the power approaches towards maximum value The $\mathrm{Q}$ factor also approaches to higher value. It means the received signals provides better quality of signal.

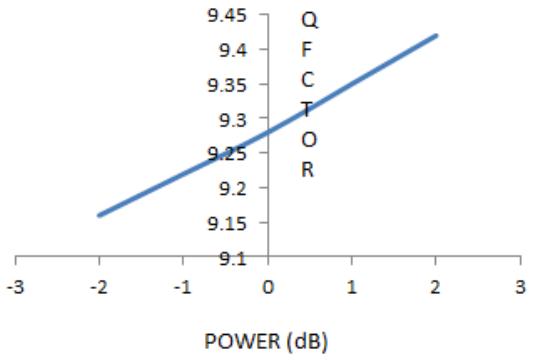

Fig 6: Q factor V/s Power(db)

\section{CONCLUSION}

In this paper, a comprehensive design in WDM PON Architecture in done. Among the impairments main focused is on FWM effect, crosstalk and try to filter its effect in the WDM PON Networks. Performance of FBG in a WDM receiver has been studied and computed results are shown by simulation as a function of number of interfering channel. A new design is developed for efficient data path provisioning with guaranteed QoT in terms of BER as well Q factor. This design is particularly very useful for high speed WDM/DWDM networks where, these impairments are high. We compare our design with design that are not impairment aware and measured the network performance in terms of blocking probability. The result shows that our crosstalk aware design reduces network blocking probability, utilizes network resources and give better quality of transmission as comparison to impairment unaware design. 


\section{ACKNOWLEDGMENTS}

This research project had been carried out in the Optical communication laboratory under the Dept. of Electronics and Communication, BIST Bhopal. All authors and co-authors are contributed in this project.

\section{REFERENCES}

[1] G. Meltz, W. W. Morey, and W. H. Glenn, "Formation of Bragg gratings in optical fibers by a transverse holographic method," Opt. Lett., vol.14, no. 15, pp. 823$825,1989$.

[2] Mizrahi "Optical properties of photosensitive fiber phase gratings," J. Light wave Technol., vol. 11, pp. 1513- 1517, Oct.1993.

[3] R. Kashyap, "Photosensitive optical fibers: Devices and applications,"Optic. Fiber Technology., vol. 1, pp. 1734, 1994.

[4] C. Elachi, "Waves in active and passive periodic structures: A review," in Proc. IEEE, vol. 64, pp. 16661698, Dec. 1976

[5] H. Kogelnik, "Coupled wave theory for thick hologram gratings," BellSyst. Tech. J., vol. 48, no. 9, pp. 29092947, 1969.

[6] M. Yamada and K. Sakuda, "Analysis of almost-periodic distributed feedback slab waveguides via a fundamental matrix approach," Appl. Opt., vol. 26, no. 16, pp. 3474$3478,1987$.

[7] H. A. Haus, Waves in Optoelectronics. Englewood[8] A. Hamakawa, T. Kato, G. Sasaki, and M. Higehara,
"Wavelength stabilization of 1.48 _m pump laser by fiber grating," in Proc. ECOC'96,Oslo, Norway, 1996, paper MoC.3.6

[8] W. J. Miniscalco, "Erbium-doped glasses for fiber amplifiers at $1500 \mathrm{~nm}, "$ J. Light wave Technol., vol. 9 , pp. 234-250, Feb. 1991.

[9] J. L. Zyskind, J. W. Sulh off, P. D. Magill, K. C. Reichmann, V. Mizrahi, and D. J. Di Giovanni, "Transmission at $2.5 \mathrm{Gbit} / \mathrm{s}$ over $654 \mathrm{~km}$ using an erbium-doped fiber grating laser source," Electron. Lett., photosensitive vol. 29, no.12, p. 1105, 1993

[10] J. T. Kringlebotn, P. R. Morkel, L. Reekie, J. L. Archam bault, and D.N. Payne, "Efficient diode-pumped single frequency erbium: ytterbium fiber laser," IEEE Photon. Technol. Lett., vol. 5, p. 1162, Oct. 1993

[11] C. R. Giles and V. Mizrahi, "Single-frequency $1559 \mathrm{~nm}$ erbium-doped fiber laser pumped by a $650 \mathrm{~nm}$ semiconductor laser," Appl. Opt., tobe published.

[12] C. R. Giles and E. Desurvire, "Modeling erbium-doped fiber amplifers,"J. Light wave Technol., vol. 9, pp. 271283, Feb. 1991.

[13] C. R. Giles, J. Stone, L. W. Stulz, K. Walker, and C. A. Burrus, "Gain enhancement in reflected-pump erbiumdoped fiber amplifiers," in Tech. Dig. Optic. Amplifiers and Their Applications, 1991, vol. 13, paperThD2, pp. $148-151$.

[14] A. A. M. Saleh, R. M. Jopson, J. D. Evankow, and J. Aspell, "Modeling of gain in erbium-doped fiber amplifiers," IEEE Photon. Technol. Lett. ,Oct. 1990. 